Ricerca in Psicoterapia / Research in Psychotherapy: Psychopathology, Process and Outcome 2009; 1-2 (12): www.spr-italia.it/pdf/spr_volume_12.pdf

\title{
Aspetti relazionali ed organizzativi delle comunità terapeutiche per adolescenti quali fattori di trattamento: validazione di uno strumento
}

Stefania Cristofanelli', Omar Fassio², Laura Ferro ${ }^{1}$, Alessandro Zennaro ${ }^{1}$

Sommario: Questo contributo intende porre il costrutto d clima organizzativo quale fattore terapeutico specifico di trattamento nei contesti residenziali di cura, contesto in cui la qualità dell'ambiente, le atmosfere emotive e l'abitare stesso diventano la materia del lavoro comunitario. In tal senso, l'équipe terapeutica-educativa diventa Lo Strumento principale di cura. L'obiettivo è dunque quello di costruire un questionario in grado di funzionare come una sorta di "termometro" per misurare lo stato di salute/malessere dell'équipe di lavoro, ponendo le basi per un successivo percorso di riflessioni e possibilità di intervento sulle criticità emerse (supervisione/formazione). Tale strumento è stato somministrato a 173 soggetti, operatori di CT per adolescenti, distribuite su tutto il territorio nazionale. Sulla base delle procedure statistiche di validazione, la versione finale risulta composta da 119 item.

Parole chiave: ambiente emotivo, ambiente organizzativo, comunità terapeutica, relazione terapeutica.

Abstract: The aim of this work is to propose a new instrument to investigate organizational characteristics in Therapeutic Communities for adolescents. "Organizational Climate" is considered as a specific therapeutic factor in a residential care treatment where the quality of the environment and the emotional atmosphere become essential aspects of communitarian work. In such a context, the working team is the main instrument of the treatment (educational and therapeutic). The questionnaire has been administered to 173 subjects, working in therapeutic communities for adolescents, searched in the whole of the Italian territory. The last version of the statistic, that has been carried out, enhanced 119 items.

Key words: emotional environment, organizational environment, therapeutic community, therapeutic relationship.

${ }^{1}$ Università della Valle d'Aosta; Tiarè Onlus, Torino; ${ }^{2}$ Università di Torino

Corrispondenza: Dr.ssa Cristofanelli Stefania, Facoltà di Psicologia, Università della Valle d'Aosta, Strda dei Cappuccini, 2/a, 11100, Aosta, s.cristofanelli@univda.it 


\subsection{Introduzione}

La riflessione sui fattori terapeutici all'interno dei contesti residenziali di cura non può non essere ricondotta nell'alveo di ricerca più ampia che fa riferimento al confronto tra l'efficacia clinica dei fattori specifici e aspecifici della relazione di cura attraverso la revisione di studi di meta-analisi su tali componenti, condotti tra il 1970 e il 1998 (Ahn e Wampold, 2001). Per fattori specifici di cura si intendono quelle componenti riconducibili ad un orientamento e ad un modello specifico di intervento rispetto ad un altro. Per quanto concerne i fattori aspecifici e trasversali di cura, sebbene siano stati descritti in letteratura in maniera differente (Karasu, 1986; Greencavage e Norcross, 1990; Weinberger, 1995), se si prende in considerazione la classificazione di Lambert (2004), possono essere ordinati in: fattori di apprendimento, fattori supportivi, fattori di azione. Gli studi di meta-analisi, in tali contesti, hanno rilevato una sorta di equivalenza rispetto all'efficacia dei differenti fattori specifici di cura, ovvero hanno dimostrato come questi ultimi non siano direttamente responsabili dell'outcome, indicando, al contrario, come i fattori aspecifici contribuiscano con effetti più significativi all'esito del trattamento. Tale contraddizione è alimentata dal fatto che diversi studi (Martin, Garske e Davis, 2000; Horvath e Bedi, 2002; Lambert e Barley, 2002) hanno indicato, inoltre, come né i fattori specifici né quelli aspecifici siano in grado di influire in modo significativo sugli esiti del trattamento. I fattori aspecifici, dunque, sembrano essere condizioni necessarie ma non sufficienti per consentire il cambiamento. Nel complesso, l'unica componente che pare, in tali studi, venire trascurata è l'interazione tra le due tipologie di fattori, la loro influenza e potenziamento reciproci (Dazzi, Lingiardi e Colli, 2006). Si ritiene, dunque, che questo lavoro possa essere letto in tale prospettiva di analisi.

La valutazione del processo terapeutico e dei suoi risultati presenta ad oggi problemi di notevole complessità e difficoltà. È, infatti, possibile rilevare tale componente critica dalla natura prevalentemente descrittiva piuttosto che teorico-esplicativa di molte analisi relative alle dinamiche interne alla comunità terapeutica e, nello specifico, relativamente ai fattori terapeutici coinvolti. In tale analisi emerge come particolarmente rilevante la funzione della qualità del clima e dell'abitare come possibili fattori di cura, parallelamente alle criticità che di volta in volta caratterizzano tali dimensioni all'interno del lavoro gruppale. Nonostante i progressi ed i risultati raggiunti dal filone di studi relativo alla valutazione dell'intervento terapeutico durante gli ultimi cinquanta anni, il focus d'interesse, che ha connotato tali lavori è stato, per lo più volto ad approfondire quasi esclusivamente la componente, per così dire "nobile", dell'intervento, ovvero la componente "psi", tralasciando spesso di considerare le dimensioni per così dire più quotidiane dell'intervento ovvero l'abitare dei pazienti ospiti di strutture residenziali, le ventiquattro ore che questi ultimi trascorrono in struttura con gli operatori e la ricchezza delle relazioni che quotidianamente consente loro di sperimentare nuovi modelli di relazione. Questo lavoro di ricerca intende prendere in considerazione, riconoscere e valorizzare la componente educativa-psicologica nei diversi aspetti che caratterizzano il suo funzionamento, sia in termini di ruolo che di quotidianità 
che, ancora, rispetto alla relazione con le altre professionalità che operano all'interno delle comunità stessa.

Costituisce, pertanto, oggetto peculiare di indagine l'analisi del clima organizzativo quale potenziale fattore terapeutico specifico all'interno dei contesti residenziali di cura. Tale costrutto teorico, prevalentemente studiato nell'ambito dei contesti organizzativi aziendali riguardo alla soddisfazione e all'efficienza dei processi organizzativi, assume una connotazione psicologica e clinica nuova in un contesto in cui la qualità dell'ambiente, delle atmosfere emotive e dell'abitare costituiscono la materia prima del lavoro comunitario. In tale contesto, l'équipe di lavoro diventa "Lo Strumento" principale di cura, responsabile della costruzione di un clima più o meno di qualità entro cui possono prendere forma e svilupparsi le quotidiane esperienze relazionali dei pazienti come in un laboratorio di sperimentazione di nuovi modelli relazionali di cui gli operatori si fanno attori partecipi. In tale ambiente sono prevalentemente gli atti a dare forma al cambiamento, secondo la felice espressione di Racamier (1997) di atti parlanti, rispetto a cui è la qualità dell'abitare che funge da catalizzatore. Tale qualità emotiva e climatica, è determinata, nello specifico, prevalentemente dalla possibilità di contenere e sciogliere il malessere, $i$ conflitti, le dinamiche collusive e regressive che sfociano spesso in forme di burnout, in cui il "contagio" della patologia prende forma in pericolose spirali distruttive tipiche di un funzionamento antiterapeutico che si esprime attraverso la specularità delle complesse dinamiche tra gruppo-équipe e gruppo-pazienti. Preservare l'équipe, in quanto strumento principe di cura, da tali tipologie di funzionamento costituisce, pertanto, oltre che un fondamentale obiettivo di lavoro, una questione etica.

Date tali premesse, occorre considerare, infine, la complessa e inafferrabile natura dei "fenomeni climatici" analizzati dalla letteratura di settore nazionale ed internazionale. Tra la fine degli anni ' 80 e la prima metà degli anni ' 90 si assiste ad una sorta di accettazione del fatto che non possa esistere un'unica definizione ed un unico modello di clima, valorizzando cioè la differenziazione delle proposte, e abbandonando l'idea di poter trovare un clima omogeneo all'interno della stessa realtà organizzativa, rafforzando sempre più l'idea di un'articolazione del concetto stesso (Quaglino, 2001). Si può dunque individuare, in modo trasversale, un chiaro percorso dalle atmosfere di gruppo lewiniane al clima organizzativo stesso in cui la "resistenza al cambiamento" costituisce una riproposizione della tematica originaria, percorso che può essere sovrapposto ed arricchito da tutti i contributi teorici che si sono focalizzati sull'analisi delle qualità dell'atmosfera e dell'ambiente anche nei contesti residenziali di cura. L'ambizione rimane, comunque, quella di trovare un'integrazione tra le componenti del clima più superficiali e quelle più profonde legate alla cultura e al "non detto" che in modo così sistematico e pervasivo sembrano influire sulle dinamiche operative quotidiane (Schneider, 1973).

Sul piano metodologico il focus di interesse diventa il fatto che non esistono indicatori di clima in grado di essere generalizzabili a tutte le realtà organizzative, ma che occorra considerare i fattori ritenuti più coerenti rispetto alla realtà oggetto di studio. 
Considerata, pertanto, l'estrema carenza, in particolare nell'ambito dei contesti di cura, di strumenti appositamente ideati per rilevare le caratteristiche del clima organizzativo, costituisce obiettivo principale del presente lavoro la costruzione di uno strumento specifico in grado di rilevare le criticità peculiari che connotano tali dinamiche, ovvero un questionario in grado di funzionare come una sorta di "termometro", al fine, cioè, di misurare le polarità dello stato di salute/malessere dell'équipe di lavoro attraverso cui, escludendo qualsiasi intento valutativo, poter porre le basi per un successivo percorso di riflessioni e possibilità di intervento per mezzo di un eventuale lavoro di supervisione/formazione all'interno delle differenti strutture comunitarie.

\subsection{Costruzione del questionario}

Il disegno di ricerca relativo al processo di formulazione e costruzione di tale strumento si è articolato in più fasi. In primo luogo si è ritenuto necessario procedere con un inquadramento storico e teorico delle dinamiche indagate, ripercorrendo il background culturale, sociale, ed ideologico che ha scandito la costituzione delle Comunità Terapeutiche (CT), dagli albori fino allo stato attuale. Ci si è soffermati, nello specifico, sui modelli ed i costrutti psicologici che le hanno connotate nel tempo, nell'ambito della tradizione inglese e americana, della tradizione francese e, infine, del panorama italiano (Vigorelli, 2006a, 2006b, 2008). Il lavoro è proseguito con una disamina di alcuni concettichiave, ovvero che cosa si intende per fattore terapeutico, funzionamento terapeutico e fenomeni comunitari all'interno dei contesti residenziali di cura, attraverso una lettura diacronica degli stessi a partire dai primi "esperimenti" che segnano il nascere di tali strutture, fino alla considerazione del panorama attuale nella sua complessità ed eterogeneità.

È stato, infine, analizzato il costrutto del clima organizzativo e di diagnosi organizzativa, sia all'interno dell'alveo dei contesti profit in cui è stato prevalentemente studiato, sia nelle sue caratterizzazioni specifiche e sfumate assunte nei contesti residenziali di cura, entro cui, tale costrutto, emerge arricchito e potenziato delle complesse dinamiche di propagazione della psicopatologia che popolano in modo profondo i vissuti quotidiani degli operatori.

A livello empirico, tale approfondimento è stato proficuo al fine di contestualizzare gli item del questionario relativamente alla cultura implicita che connota tali realtà terapeutiche.

Il disegno di ricerca si esplica in una fase successiva attraverso l'analisi degli strumenti di rilevazione di clima esistenti in letteratura in grado di soddisfare i requisiti ed i presupposti teorici descritti precedentemente. Di tali strumenti, è stato individuato come riferimento il Majer-D'Amato Organizational Questionnaire (M-DOQ, Majer e D'Amato, 2001), a partire dal quale, dopo un'attenta contestualizzazione degli item, ne sono stati selezionati alcuni più salienti che sono stati successivamente integrati con un'ulteriore serie di item: in parte estrapolati dall'analisi qualitativa dei trascritti di alcuni Focus Group condotti con operatori che lavorano in CT; in parte ricavati da un contributo di consulenza clinica; in parte, infine, selezionati da altre scale già disponibili in letteratura per rilevare le dinamiche di gruppo. 


\subsection{Majer-D'Amato Organizational Questionnaire}

Il Majer-D'Amato Organizational Questionnaire (M-DOQ, 2001) si presenta come un questionario multidimensionale, costituito da 120 item con rilevazione di tipo scala Likert a 4 punti in grado di fornire una stima differenziata delle componenti principali del costrutto del clima in ambito aziendale. La struttura fattoriale si articola in 13 fattori: team, leadership, job involvement, autonomia, libertà, coerenza, dinamismo, job description, equità, sviluppo, comunicazione, environment e incentivazione.

Tale strumento, inoltre si pregia di un impianto sofisticato in termini di verifica della validità e dell'attendibilità attraverso una standardizzazione italiana che ha previsto un campionamento di organizzazioni di tipo aziendale dislocate su tutto il territorio nazionale (in particolare nel Nord e Centro Italia) costituite da soggetti appartenenti a molteplici settori di organizzazioni differenti (Quaglino, 2001). La versione attuale del questionario è stata somministrata a 1837 soggetti. Può, dunque, essere definito come uno strumento valido da un punto di vista scientifico, snello nella sua utilizzabilità empirica e adatto alle diverse culture organizzative.

\subsection{Focus Group}

Tenendo come riferimento tale strumento, al fine di ampliare il processo di contestualizzazione degli item del questionario, sono stati condotti una serie di Focus Group con i coordinatori e gli educatori esperti di alcune CT. I focus group si sono articolati sull'approfondimento della tematica centrale della definizione del concetto di clima organizzativo all'interno della realtà operativa delle CT al fine di declinare il più possibile il concetto di clima nelle singole componenti che lo qualificano in modo specifico. In particolare è stato richiesto di riferire alcuni momenti di lavoro vissuti in prima persona dagli operatori che sono risultati significativi relativamente alla percezione di un clima organizzativo adeguato o non adeguato rispetto alla richieste contestuali del setting comunitario, nel tentativo di esplicitare le problematiche ed i vissuti ad esso inerenti e le eventuali ricadute terapeutiche sui pazienti (Cortese, 2004). Tutti i focus group sono stati audio-registrati e sbobinati al fine di estrapolare alcuni item da includere nella versione finale del questionario da somministrare. I gruppi erano composti da un minimo di cinque ad un massimo di sette soggetti, suddivisi in modo eterogeneo per età, sesso, e anni di lavoro presso la struttura di pertinenza. Affrontare tali tematiche attraverso le modalità di rilevazione sopra descritte, ha consentito di creare un ambiente sufficientemente libero e non saturo al fine di fare emergere criticità specifiche per il contesto di lavoro indagato, contribuendo a dare un senso all'oggetto di ricerca in modo collettivo e producendo una versione della realtà nel tentativo preciso di comprenderla, partendo dall'idea che "un'organizzazione non è un qualcosa di dato, ma deriva dalle rappresentazioni che i diversi attori coinvolti hanno di essa" (Masoni, Balducci, Coratti, Hansen e Melgiovanni, 2000). 
Il materiale ottenuto è stato elaborato attraverso l'applicativo informatico Atlas.ti, all'interno dell'inquadramento teorico della Ground Theory e successivamente per mezzo dell'applicativo Monoconc Pro al fine di estrarre le occorrenze dei lemmi che sono comparsi con una frequenza più elevata all'interno dei differenti trascritti. Dall'analisi dei verbatim sono emersi alcuni temi principali tra cui: criticità relative al turnover degli operatori; problemi rispetto al carico eccessivo; problematiche relative all'inserimento di nuovi pazienti; difficoltà a tradurre nella quotidianità il progetto terapeutico complessivo; criticità nella gestione dei momenti di crisi; problematiche rispetto al ruolo; criticità specifiche relative alla psicopatologia dei pazienti, da cui sono stati estrapolati tre fattori: "criticità organizzative relative alla struttura", "criticità organizzative relative alla psicopatologia" e "professionalità" (vedi tabella 1).

\subsection{Item componente clinica}

In seconda battuta alcuni item sono stati formulati sulla base di una consulenza fornita dall'estensore clinico del Progetto Minori all'interno dell'Associazione di Servizi per la Salute Mentale Tiarè onlus. Da questa componente sono stati estrapolati due fattori: "chiarezza ed efficacia della collaborazione tra equipe e clinici" e "criticità dei rapporti con i servizi" (vedi tabella 1).

\subsection{Altre Scale}

Attraverso un'accurata analisi della letteratura nazionale e internazionale relativa a strumenti di analisi affini ai nostri interessi in questo settore, sono state individuate alcune scale da cui sono stati selezionati gli item che potevano essere integrati in seguito ad un'attenta contestualizzazione e traduzione. Di seguito verranno presentate le fonti da cui sono stati estratti gli item della versione del questionario somministrata:

1QUESTIONARIO SUL CLIMA DI GRUPPO (MacKenzie, 1981; traduzione a cura della Prof.ssa Francesca Giannone, Università degli studi di Palermo, utilizzato all'interno di comunità educative).

2COHESION SCALE (Piper, 1983; traduzione nostra).

3BASIC NEEDS SATISFACTION AT WORK SCALE (Deci, Ryan, Gagné, Leone, Usunov e Kornazheva, 2001; traduzione nostra).

4MCDERMOTT BURNOUT INVENTORY (McDermott, 1984; tratto da Majer e D'Amato, 2001)

5RELATIONSHIP QUESTIONNAIRE (Bartholomew \& Horowitz, 1991). 
Da tali scale sono stati ricavati quattro fattori: "problemi nella disponibilità alla relazione", "soddisfazione sul lavoro", "paura, pesantezza e assenza di investimento", "collaborazione e disponibilità tra colleghi" (vedi tabella 1).

\subsection{Accordo Inter-Giudice}

La prima versione del questionario, ottenuta dalla giustapposizione degli item provenienti dagli strumenti sopra esposti, si compone di 144 item

Successivamente si è proceduto con la valutazione dell'accordo intergiudice relativamente all'attribuzione di ogni singolo item rispetto alla struttura fattoriale del questionario di Mayer e D'Amato. I 144 item ottenuti sono stati sottoposti alla valutazione da parte di 7 giudici esperti con la consegna di lavorare individualmente per ricondurre ciascun item ai 13 fattori caratterizzanti la struttura fattoriale dell'M-DOQ, segnalando eventualmente gli item che non potevano essere ricondotti a nessun fattore all'interno della categoria "altro". Considerata la presenza di un considerevole numero di item aggiuntivi rispetto a quelli ricavati dall'M-DOQ, l'accordo intergiudice non è risultato elevato, seppur adeguato per quanto concerne $\mathrm{i}$ fattori previsti da Mayer. Utilizzando l' indice di concordanza per variabili categoriali Kappa di Cohen (Cohen, 1960), i risultati ottenuti dai confronti a coppie hanno confermato un'adeguata attendibilità delle ricodifiche $(0.300<K$ di Cohen $<$ $0.536 ; p<.001)$.

In tal senso è parso plausibile prevedere una struttura dello strumento finale riconducibile ad un numero superiore di fattori. Prima della somministrazione definitiva, la versione finale del questionario è stata sottoposta, infine, ad una revisione da parte di 5 operatori psicologi al fine di verificare la presenza di eventuali errori nella formulazione degli item stessi (a livello semantico, sintattico e ortografico) che avrebbero potuto andare a discapito della comprensione finale dello strumento.

\subsection{Fattori totali estrapolati}

In totale, oltre ai fattori previsti dall'M-DOQ di Mayer e D'Amato, sono stati analizzati i seguenti fattori:

Tabella 1. Fattori estrapolati dai Focus group, dalla consulenza dei clinici e da altre scale disponibili in letteratura 
Ricerca in Psicoterapia / Research in Psychotherapy: Psychopathology, Process and Outcome 2009; 1-2 (12): www.spr-italia.it/pdf/spr_volume_12.pdf

\begin{tabular}{|c|c|c|c|}
\hline FATTOFE & FONIE & FATTORE & FONIE \\
\hline $\begin{array}{l}\text { OHAREZAed甲TCAOAdella } \\
\text { COLABCRAZONEtraEQUPEE } \\
\text { QUNG (8item) }\end{array}$ & $\begin{array}{l}\text { ITEM } \\
\text { COMPONENIE } \\
\text { OINCA }\end{array}$ & $\begin{array}{l}\text { PROBEM NBШADISPONBIUTA' } \\
\text { AШAREAZONE(4item) }\end{array}$ & $\begin{array}{l}\text { PEATTONGHP } \\
\text { QESTIONAIFE }\end{array}$ \\
\hline $\begin{array}{l}\text { CRTIATA' RAPPCRII œOn ISRMZ } \\
\text { (3item) }\end{array}$ & $\begin{array}{l}\text { ITEM } \\
\text { CONPONENIE } \\
\text { CINCA }\end{array}$ & $\begin{array}{l}\text { SOSDSFAZONESULAVCRO } \\
\text { (3item) }\end{array}$ & COESONSCALE \\
\hline $\begin{array}{l}\text { ORTIATA' CRGANZZATIVE } \\
\text { R日ATIVEALASTRUTIURA } \\
\text { (6item) }\end{array}$ & FOASCRAP & $\begin{array}{l}\text { PALREPESANIEZAAEASSENAAd } \\
\text { INMESTIMENTO(9item) }\end{array}$ & $\begin{array}{l}\text { MODERDTTBUANOUT } \\
\text { IMENTCRY } \\
\text { BASICNETSSATISFACTION } \\
\text { AT WOFKSCALE }\end{array}$ \\
\hline $\begin{array}{l}\text { CRTIATA' CRGANZZATIVE } \\
\text { REATIVEAШAPSIOOPATOLOAA } \\
\text { (6item) }\end{array}$ & FOASGAOP & $\begin{array}{l}\text { @Q } А \text { ABORAZONEEDSPONBUTA' } \\
\text { PACQUEGH (3item) }\end{array}$ & $\begin{array}{l}\text { QESTIONAROSU OMMAI } \\
\text { GAPPO }\end{array}$ \\
\hline $\begin{array}{l}\text { PROFSSIONAUTA' } \\
\text { (4item) }\end{array}$ & FOASGAOP & & \\
\hline
\end{tabular}

\section{Materiali e Metodi}

La metodologia di costruzione del questionario si articola attraverso una pre-ricerca esplorativa.

La procedura di reclutamento dei soggetti e delle strutture da coinvolgere per la somministrazione del questionario ha costituito motivo di empasse per il presente lavoro, se si considera che non esiste, a livello nazionale, un elenco ufficiale delle strutture terapeutiche residenziali per minori, che in alcuni casi decidono di "non svelarsi". Tale "ostacolo" è stato, del resto, per lo più condiviso con alcuni dei referenti delle strutture stesse che hanno partecipato al progetto, nella misura in cui è stata espressa la sensazione di lavorare "in solitudine" ovvero di non poter contare o fare riferimento ad un modello a rete più esplicito che coinvolga tutte le strutture presenti sul territorio nazionale. Per alcune realtà esiste la necessità di non essere accessibili in quanto strutture protette, considerata l'età degli ospiti e le dinamiche familiari fortemente compromesse. Rimane il fatto che tra professionisti del settore, talvolta, emerge il vissuto di "isolamento" che condiziona le possibilità/potenzialità di intervento.

\subsection{Campione}

Complessivamente il gruppo preso in esame è costituito da 173 soggetti diversificati per profili professionali ed accomunati dal fatto di lavorare all'interno di CT per adolescenti dislocate in modo non omogeneo sul territorio nazionale (Piemonte, Lombardia, Liguria, Emilia-Romagna, Toscana, Marche, Abruzzo, Lazio, Campania). 
Ricerca in Psicoterapia / Research in Psychotherapy: Psychopathology, Process and Outcome 2009; 1-2 (12): www.spr-italia.it/pdf/spr_volume_12.pdf

Tabella 2. Variabili socio anagrafiche del campione della ricerca

\begin{tabular}{|c|c|c|}
\hline Categorie & & $\% / n$ \\
\hline Sesso operatori & $\begin{array}{l}\text { Maschi } \\
\text { femmine }\end{array}$ & $\begin{array}{l}39.1 \% \\
60.9 \%\end{array}$ \\
\hline Titolo di studio & $\begin{array}{l}\text { Diploma scuola media inferiore } \\
\text { Diploma scuola media superiore } \\
\text { Corso di formazione post diploma } \\
\text { Laurea } \\
\text { Post laurea }\end{array}$ & $\begin{array}{l}2 \% \\
11 \% \\
12 \% \\
50 \% \\
25 \%\end{array}$ \\
\hline Ruolo & $\begin{array}{l}\text { Educatori } \\
\text { Operatori } \\
\text { Coordinatori } \\
\text { Psicologi } \\
\text { Operatori Socio Sanitari } \\
\text { Psichiatri } \\
\text { Psicoterapeuti } \\
\text { Psicopedagogisti } \\
\text { Tirocinanti volontari }\end{array}$ & $\begin{array}{l}50 \% \\
20 \% \\
11 \% \\
10 \% \\
4 \% \\
1.16 \% \\
1,16 \% \\
1,16 \% \\
1,16 \%\end{array}$ \\
\hline Nome comunità & $\begin{array}{l}\text { Asso } \\
\text { Cavanà } \\
\text { Crisalide } \\
\text { Eimì } \\
\text { Il Delfino } \\
\text { Il Faro } \\
\text { Il Porto } \\
\text { L'Imprevisto } \\
\text { La Soffitta } \\
\text { Liberitutti } \\
\text { Passaggi } \\
\text { *** } \\
\text { Rosa dei Venti } \\
\text { San Maurizio } \\
\text { Sogni di loro } \\
\text { UONPIA Desio } \\
\text { Capena } \\
\text { Mondo piccolo } \\
\text { Tuga }\end{array}$ & $\begin{array}{l}17 \\
11 \\
7 \\
11 \\
6 \\
6 \\
20 \\
15 \\
7 \\
8 \\
13 \\
2 \\
5 \\
9 \\
6 \\
5 \\
14 \\
6 \\
5\end{array}$ \\
\hline
\end{tabular}

Nota: la struttura contrassegnata $\mathrm{da}^{* * *}$ ha richiesto esplicitamente di mantenere l'anonimato

Il campione è stato descritto sulla base delle caratteristiche socioanagrafiche (vedi tabella 2) che sono state tenute in considerazione per le successive analisi statistiche. Per quanto riguarda la variabile sesso, il campione è costituito da un $60,9 \%$ di donne e da un $39,1 \%$ di uomini. Per quanto concerne la variabile titolo di studio, il campione possiede un livello di scolarizzazione medio-alto, se si considera che 1 ' $11 \%$ possiede un diploma di scuola Media Superiore, il 12\% dispone di corsi di formazione post diploma, ed in particolare il $50 \%$ ha conseguito la Laurea insieme ad un $25 \%$ che ha un livello di formazione post-universitaria; il restante $2 \%$, infine, è in possesso del diploma di scuola Media Inferiore. Rispetto alla variabile ruolo, considerata l'eterogeneità delle diciture rilevate dalla compilazione della parte anagrafica 
del questionario, le differenti definizioni sono state raggruppate in 7 categorie. Il campione si caratterizza per una prevalenza di profili educativi, ovvero si compone per il $50 \%$ di educatori e per il $20 \%$ di operatori; la restante parte si suddivide tra un $11 \%$ di soggetti costituito da coordinatori, un $10 \%$ di psicologi, un $4 \%$ di operatori socio sanitari (OSS), un $1.16 \%$ di psichiatri, un $1.16 \%$ di psicoterapeuti, un $1.16 \%$ di psicopedagogisti ed infine un $1.16 \%$ tra tirocinanti e volontari. Complessivamente l'età media del campione è di 34,2 anni (min = 21; Max $=56 ;$ Dev. St. = 7.64). Mediamente i soggetti hanno dichiarato di lavorare nella comunità di pertinenza da circa 4 anni, con un valore minimo di un mese ed un valore massimo di 20 anni, mentre, per quanto riguarda il lavoro in contesti di équipe in generale, il valore medio indicato è stato di circa 6 anni e mezzo, con un valore minimo di un mese ed un valore massimo di 20 anni.

\section{Analisi dei risultati}

Le procedure statistiche di validazione preliminare dello strumento hanno compreso analisi descrittive, analisi fattoriali e analisi dell'affidabilità interna (alpha di Cronbach) dei singoli item del questionario e delle scale di fattori proposte. Le analisi fattoriali sono state condotte utilizzando il metodo Unweighted Least Squares, ponendo il loading a .40, e considerando come criterio di scelta per il numero dei fattori lo Scree Test di Cattel con eigenvalue $>1$.

Sulla base della lettura incrociata dei dati delle precedenti analisi, si è proceduto, con l'eliminazione degli item (vedi tabella 3 ) che non soddisfacevano i rispettivi requisiti. La struttura fattoriale finale del questionario prevede 22 fattori articolati (vedi tabella 3 ).

Il successivo livello di analisi è stato lo studio delle correlazioni tra i punteggi fattoriali individuati. Da queste è risultato che $\mathrm{i}$ fattori appaiono essere complessivamente sufficientemente ortogonali, pur sovrapponendosi in parte rispetto ad alcune dimensioni. Le correlazioni tra i fattori sono le seguenti: "criticità organizzative patologia" con "coerenza" $(r=.210)$, con "incentivazione" $(r=-.233)$ e con "sviluppo" $(r=.190)$; "chiarezza efficacia collaborazione equipe-clinici" con "job involvement" $(r=-.185)$; "problemi disponibilità alla relazione" con "coerenza" $(r=.178)$ ed infine "leadership" con "sviluppo" $(r=-.230)$.

Sulla scorta delle analisi statistiche effettuate emerge una versione definitiva dello strumento costituito da 119 item (l'elenco completo di questi item viene riportato nell'Appendice finale). 
Ricerca in Psicoterapia / Research in Psychotherapy: Psychopathology, Process and Outcome 2009; 1-2 (12): www.spr-italia.it/pdf/spr_volume_12.pdf

Tabella 3. Elenco dei 22 fattori, delle Alpha di Cronbach e degli item eliminati

\begin{tabular}{|c|c|c|}
\hline FATTORE & $\alpha$ & Numero Item \\
\hline $\begin{array}{l}\text { 1. CHIAREZZA EFFICACIA } \\
\text { COLLAB. EQUIPE E CLINICI }\end{array}$ & 0.772 & $1,4,3,6,2,5,7 *, 8 * *, 9 *, 12,14$ \\
\hline $\begin{array}{l}\text { 2. CRITICITA' RAPPORTI } \\
\text { SERVIZI }\end{array}$ & 0.608 & $10 * *, 11 * *, 13 *, 16,15,17$ \\
\hline $\begin{array}{l}\text { 3. CRITICITA' } \\
\text { ORGANIZZATIVE } \\
\text { STRUTTURA }\end{array}$ & 0.712 & $135,126,141,129 * *, 133 *, 143,139,136$ \\
\hline $\begin{array}{l}\text { 4. CRITICITA' } \\
\text { ORGANIZZATIVE } \\
\text { PSICOPATOLOGIA }\end{array}$ & 0.677 & $144,132,137,142,127,134,138 *$ \\
\hline 5. PROFESSIONALITA' & 0.349 & $34,128,130,131 * * *, 140 * *$ \\
\hline $\begin{array}{l}\text { 6. PROBLEMI } \\
\text { DISPONIBILITA' ALLA } \\
\text { RELAZIONE }\end{array}$ & 0.173 & $36, \mathbf{3 7 * *}, \mathbf{3 8 *}, 39,40,41, \mathbf{4 2 * *}$ \\
\hline $\begin{array}{l}\text { 7. PAURE PESANTEZZA E } \\
\text { ASSENZA INVESTIMENTO }\end{array}$ & 0.795 & $\begin{array}{l}28,32, \mathbf{3 3} *, \mathbf{3 1} *, 123,26, \mathbf{3 5 * *}, \mathbf{2 7} * *, 124,24,29, \\
30,25, \mathbf{1 2 5} *\end{array}$ \\
\hline $\begin{array}{l}\text { 8. SODDISFAZIONE SUL } \\
\text { LAVORO }\end{array}$ & 0.434 & $18,19,20$ \\
\hline $\begin{array}{l}\text { 9. COLLABORAZIONE E } \\
\text { DISPONIBILITA' FRA } \\
\text { COLLEGHI }\end{array}$ & 0.665 & $21,22,23$ \\
\hline 10. COERENZA & 0.746 & $43,56,75,81,115 *$ \\
\hline 11. INCENTIVAZIONE & 0.253 & $44,64, \mathbf{8 5} * * *, 116$ \\
\hline 12. JOB DESCRIPTION & 0.779 & $83,107,88,45,70,97$ \\
\hline 13. LEADERSHIP & 0.899 & $\begin{array}{l}104,109,73,69,51,113,59,106,46,95,50,86,101, \\
66,52,48\end{array}$ \\
\hline 14. COESIONE DI GRUPPO & 0.825 & $53,47,57,90,120,61,111$ \\
\hline 15. COMUNICAZIONE & 0.543 & $99,54,76,77,93,103,96$ \\
\hline 16. JOB INVOLVEMENT & 0.653 & $55,112,58,116 *, 117,119,79$ \\
\hline 17. AUTONOMIA & 0.141 & $60,92,80,87,65$ \\
\hline 18. LIBERTA' & -0.369 & $62,78,82,91,114,98$ \\
\hline 19. DINAMISMO & 0.619 & $108,72,94,102,63,105 * *$ \\
\hline 20. ENVIRONMENT & 0.590 & $110,67,68$ \\
\hline 21. EQUITA' & 0.739 & $71,74,113,89,118$ \\
\hline 22. SVILUPPO & 0.98 & $64^{*}, 122,84,121,100 * * *$ \\
\hline
\end{tabular}

Note:

- gli item indicati con * sono stati eliminati poichè hanno evidenziato una varianza ridotta (range di risposta: $2-4$ o $1-3$ );

- gli item indicati con ** sono stati eliminati poichè hanno presentato un numero di missing tra 15 e 21 ;

- gli item indicati con *** sono stati eliminati poiché, caratterizzati da scarse caratteristiche psicometriche sulla base delle informazioni provenienti dalle Analisi Fattoriali e dal calcolo dell'Alpha di Cronbach.

\section{Discussione e conclusioni}

L'obiettivo finale del lavoro si è composto di due finalità complementari: da un lato la predisposizione di uno strumento specifico, teoricamente e psicometricamente affidabile ai fini dell'indagine del clima organizzativo all'interno delle CT, dall'altro la verifica dello stesso, quale strumento di rilevazione in progress degli elementi che connotano e definiscono tale costrutto, attraverso una pre-ricerca limitata e del tutto preliminare. 
Il lavoro svolto ha prodotto come risultato operativo la disponibilità della versione finale di un questionario di rilevazione, fruibile ai fini dell'indagine del clima organizzativo, nelle sue differenti articolazioni, all'interno delle CT. Lo strumento individuato andrà, quindi, successivamente validato, secondo le procedure già presentate, allo scopo di delinearne le caratteristiche psicometriche essenziali per procedere ad un suo utilizzo su più ampia scala. I dati provenienti dalla pre-ricerca condotta appaiono confortanti in merito alla capacità dello strumento di indagare gli aspetti di funzionamento delle équipe educative all'interno dei contesti terapeutici (coverage). Esso appare altresì esaustivo delle diverse componenti di significato implicate nel costrutto.

I limiti del presente lavoro sono, in parte, riconducibili alla complessità e "inafferrabilità" del costrutto indagato, ampiamente denunciata dalla letteratura di settore, il quale implica il tenere in considerazione un modello di rilevazione multidimensionale che si esplica attraverso una struttura fattoriale che contempla numerose dimensioni ( 22 fattori). Le procedure statistiche di validazione preliminare dello strumento, inoltre, sono state applicate ad un gruppo di soggetti con una numerosità relativamente limitata (173 soggetti), pur corrispondendo alla quasi totalità degli operatori che lavorano all'interno delle strutture terapeutiche per adolescenti presenti sul territorio nazionale, limite imposto, pertanto, dalla situazione attuale relativa alla realtà delle Comunità Terapeutiche per adolescenti e dal tentativo di applicare tale metodologia di analisi ad un contesto reale di cura. All'interno della scheda anagrafica, sulla base dei risultati della prima somministrazione, ci riproponiamo, per una futura versione, di raggruppare le descrizioni dei profili degli operatori in categorie discrete al fine di limitare l'estrema eterogeneità dei profili emersi dalla compilazione degli stessi, difficile da gestire per le analisi successive. Tale strategia è stata, infatti, adottata già nella fase di elaborazione preliminare del presente lavoro, raggruppando i profili in 9 categorie. Infine, una componente perfettibile è da individuarsi a livello della numerosità stessa degli item che, nella versione somministrata, costituita da 144 item, ha potuto determinare un potenziale bias di attenzione e capacità di concentrazione da parte degli operatori, appesantendo, talvolta, la comprensione degli item stessi, andando chiaramente ad influire sui tempi di compilazione del questionario. $\mathrm{Ci}$ proponiamo, pertanto, di ridurre tale componente attraverso la presentazione della versione definitiva dello strumento composta da 119 item (vedi Appendice finale).

Sarà nostro obiettivo, a questo punto, proseguire il lavoro impostato attraverso un'applicazione sistematica dell'inventario ad un campione più vasto di operatori che lavorano all'interno di CT, finalizzata ad una sua validazione definitiva e, soprattutto, alla sua implementazione all'interno delle routine di ricerca attive nei contesti residenziali di cura. La valutazione dell'influenza del costrutto di clima all'interno dei possibili fattori terapeutici operanti in tali servizi potrebbe infatti costituirsi quale importante elemento di innovazione rispetto alla ricerca ma anche alla clinica. Una clinica che possa riconoscere oltre ai tradizionali fattori specifici, anche l'importanza della quotidianità relazionale quale possibile fattore interveniente nella modificazione comportamentale e strutturale dei pazienti trattati presso tali contesti. Auspichiamo, dunque, che 
questo possa costituire un ulteriore passo nella direzione di avvicinare la ricerca alla pratica clinica nei contesti reali in cui questa viene esercitata.

\section{Bibliografia}

Ahn, H., \& Wampold B. E. (2001). Where are the specific ingredients? A meta-analysis of component studies in counseling and psychoterapy. Journal of Counseling Psychology, 48, 251-257.

Bartholomew, K., \& Horowitz L. M. (1991). Attachment styles among young adults: A test of a four-category model. Journal of Personality and Social Psychology, 61, 226-244.

Cohen, J. (1960). A coefficient of agreement for nominal scales. Educational and Psychological Measurement, 20, 37-46.

Cortese, C. G. (2004). La soddisfazione per il lavoro. Sviluppo \& Organizzazione, 206, 89-104.

Corulli, M. (a cura di) (1997). Terapeutico e antiterapeutico. Cosa accade nelle comunità terapeutiche? Torino: Bollati Boringhieri.

Cristofanelli, S., \& Zennaro, A. (2007). Possibili evoluzioni del CS: la valutazione dei contenuti. In Zennaro A., Lis A., Salcuni S., Parolin L., Mazzeschi C. Il Rorschach secondo il Sistema Comprensivo di Exner. Manuale per l'utilizzo dello strumento. Milano: Raffaello Cortina.

D'Amato, A., \& Majer, V. (2005). Il vantaggio del clima. La ricerca del clima per lo sviluppo organizzativo. Milano: Raffaello Cortina Editore.

Dazzi, N., Lingiardi, V., \& Colli A. (2006). La ricerca in psicoterapia. Modelli e strumenti. Milano: Raffaello Cortina Editore.

Deci E. L., Ryan R. M., Gagne M., Leone D. R., Usunov, J., \& Kornazheva, B. P. (2001). Need satisfaction, motivation, and well-being in the work organisations of a former eastern bloc country: A cross-cultural study of self-determination. Personality and Social Psychology Bulletin, 27, 930-942.

De Coro, A., \& Andreassi, S. (2004). La ricerca empirica in psicoterapia. Roma: Carocci.

Ferruta, A., Foresti, G., Pedriali E., \& Vigorelli M. (a cura di) (1998). La comunità terapeutica. Tra mito e realtà. Milano: Raffaello Cortina Editore.

Giammaria, M., \& Zennaro, A. (in stampa). La comunità terapeutica per adolescenti. Descrizione di un modello. Roma: Carocci.

Glisson, C. (2002). The Organizational Context of Children's Mental Healh Services. Clinical Child and Family Review, 5, 233-253

Haigh, R. (2002). Therapeutic community research: past, present and future. Psychiatric Bullettin, 26, 65-68.

Horvath, A.O., \& Bedi R.P. (2002). The alliance. In J. Norcross (a cura di), Psychotherapy Relations That Work. Oxford: Oxford University Press.

Johannesson, R. E. (1976). Some problems in the Measurement of Organizational Climate. Administrative Science Quarterly, 21, 95-103.

Karasu, T. B. (1986). Il dilemma tra specificità e non specificità: verso l'identificazione dei fattori di cambiamento terapeutico. Tr. it. in Del Corno F., Lang M. (a cura di), Psicologia Clinica, (1) Fondamenti storici e metodologici. Milano: Franco Angeli. 
Kressel, D., De Leon, G., Palij, M., \& Rubin, G. (2000). Measuring client clinical progress in therapeutic community treatment. The Therapeutic Community Client Assessment Inventory, Client Assessment Summary, and Staff Assessment Summary (2000). Journal of Substance Abuse Treatment, 19, 267-272.

Lambert, M. J., (a cura di) (2004). Bergin and Garfield's Handbook of Psychotherapy and Behavior Ch'ange, 5th ed. New York: John Wiley and Sons.

Lis, A., Zennaro, A., Parolin, L., \& Salcuni S. (2007). Rorschach Comprehensive System data for a sample of 249 adult not patients from italy. Journal of Personality Assessment, vol. 89, 80-84.

Lombardo, A. (2004). La comunità psicoterapeutica. Cultura, strumenti, tecnica. Milano: Franco Angeli.

Martin, D. J., Garske, J. P., \& Davis, M. K. (2000). Relationof the therapeutic alliance with outcome and other variables: A meta-analytic review. Journal of Counseling and Clinical Psychology, 68, 438-450.

Majer, V., \& D'Amato, A. (2001). Majer-D'Amato Organizational Questionnaire (M-DOQ).Questionario Multidimensionale del Clima Organizzativo. Padova: Unipress.

MacKenzie, D. A. (1981). Statistics in Britain, 1865-1930: The Social Construction of Scientific Knowledge. Edinburgh: Edinburgh UP.

Masoni, P., Balducci, P., Coratti, C., Hansen, P., \& Melgiovanni S. (a cura di), (2000). Le Comunità Terapeutiche. Ricerca sul funzionamento organizzativo e formazione degli operatori. Roma: Edizioni Kappa.

Pedriali, E. (2000). La professionalità dell'Operatore di Comunità: tra funzione psicoterapeutica e funzione psicoeducativa. In L. Dozza (a cura di) Professioni educative per il Sociale. Teorie e metodologie del lavoro di gruppo. Bari: Adda Editore.

Perini, M. (2002). La cultura organizzativa della comunità terapeutica. Disponibile online in http://www.psychomedia.it/pm-proc/duparc2002/perini.htm

Piper, W. E. (1983). Cohesion as a Basic Bond in Groups. Human Relations, 36, 93-108.

Quaglino, G. P. (2001). In V. Majer e A. D'Amato, Majer-D'Amato Organizational Questionnaire (M-DOQ).Questionario Multidimensionale del Clima Organizzativo. Padova: Unipress.

Quaglino, G. P. (2004). La vita organizzativa. Difese, collusioni e ostilità nelle relazioni di lavoro. Milano: Raffaello Cortina Edtore.

Quaglino, G. P., Mander, M. (1987). Climi organizzativi. Bologna: Il Mulino.

Racamier, P. C. (1997). Una comunità di cura psicoterapica. Psichiatrie Francaise, 1, 137-152.

Rapoport, R. N. (1960). Community as a Doctor. New Perspectives on a Therapeutic Community. London: Tavistock Publications.

Schneider, B. (1973). The perception of organizational climate: the customer's view. Journal of Applied Psychology, 57, 248-256.

Vigorelli, M. (2006). La Comunità Terapeutica come organizzazione sociale per la cura. Il Modello della Comunità Terapeutica: L'area inglese. Terapia di Comunità, 6,32. 
Vigorelli, M. (2006). La Comunità Terapeutica come organizzazione sociale per la cura. Il Modello della Comunità Terapeutica: L'area statunitense. Terapia di Comunità, 6, 34 .

Vigorelli, M. (2008). La Comunità Terapeutica come organizzazione sociale per la cura. Il Modello della Comunità Terapeutica: L'area francese. Terapia di Comunità, 8, 38.

Zennaro, A., Cristofanelli, S., \& Vottero Ris, F. (2006). Indicazioni per una buona metodologia della ricerca. In N. Dazzi, V. Lingiardi, A. Colli, La ricerca in psicoterapia. Modelli e strumenti. Milano: Raffaello Cortina.

Zennaro, A., \& Lis, A. (2006). L'uso dei test proiettivi nella ricerca in psicoterapia: vantaggi e limiti. In: Dazzi N., Lingiardi V., Colli A.. La ricerca in Psicoterapia. Modelli e strumenti. Milano: Raffaello Cortina.

Zennaro, A., Cristofanelli, S., Ferro, L., \& Vottero Ris F. (2007). Problemi legati alla diagnosi di personalità nell'età adulta e in adolescenza. Psicologia Clinica dello Sviluppo, 1, 142-172.

Zennaro, A., \& Ferro L. (2007). L'utilizzo del Rorschach nel contesto giuridico-forense. In Zennaro A., Lis A., Salcuni S., Parolin L., Mazzeschi C. Il Rorschach secondo il Sistema Comprensivo di Exner. Manuale per l'utilizzo dello strumento. Milano: Raffaello Cortina.

Zennaro, A., Ferro, L., Salcuni, S., \& Tenzon, S. (2007). Confronto fra somministrazione individuale e collettiva del test di Rorschach: primi dati. Bollettino di Psicologia Applicata, vol. 253, 70-81.

Zennaro, A., Lis, A., Salcuni, S., Parolin, L., \& Mazzeschi, C. (2007). Il Rorschach secondo il Sistema Comprensivo di Exner. Manuale per l'utilizzo dello strumento. Milano: Raffaello Cortina.

Zennaro, A., Parolin, L., Calvo, V., \& Meyer, G. (2007). The impact of administration and inquiry on Rorschach Comprehensive System protocols in a national reference sample. Journal of Personality Assessment, vol. 89; p. 193-200.

Zennaro, A., Cristofanelli, S., Ercolin, D. M., Ferro, L., \& Vottero Ris F. (2008). Gli inventari di personalità di Millon: Millon Clinical Multiaxial Inventory (MCMI III) e Millon Adolescent Clinical Inventory (MACI). Basi teoriche, descrizione e uso clinico. In Lang M \& Del Corno F. (Eds). Linee di ricerca in testologia. Milano: Franco Angeli.

Zennaro, A., Ferracuti, S., Lang, M., \& Sanavio E. (2008). MCMI III Millon Multiaxial Clinical Inventory: Adattamento Italiano. Firenze: Giunti OS.

Ricevuto: 2 dicembre 2008. Revisionato: 28 giugno 2009. Accettato: 13 luglio 2009. 
Ricerca in Psicoterapia / Research in Psychotherapy: Psychopathology, Process and Outcome 2009; 1-2 (12): www.spr-italia.it/pdf/spr_volume_12.pdf

APPENDICE: Media e deviazione standard degli item totali (prima versione)

\begin{tabular}{|c|c|c|c|c|c|c|c|c|}
\hline ITEM & M & DS & ITEM & M & DS & ITEM & M & DS \\
\hline 1 & 2.97 & 0.845 & 51 & 1.05 & 1.016 & 101 & 1.87 & 0.912 \\
\hline 2 & 2.65 & 0.856 & 52 & 1.01 & 0.975 & 102 & 1.9 & 0.875 \\
\hline 3 & 1.98 & 0.979 & 53 & 1.39 & 0.835 & 103 & 2.19 & 1.016 \\
\hline 4 & 3.21 & 0.818 & 54 & 1.97 & 0.881 & 104 & 1.88 & 0.889 \\
\hline 5 & 2.07 & 0.964 & 55 & 3.09 & 0.858 & 105 & 3.39 & 0.729 \\
\hline 6 & 3.65 & 0.583 & 56 & 1.73 & 0.946 & 106 & 2.07 & 0.939 \\
\hline 7 & 3.17 & 0.73 & 57 & 1.63 & 0.833 & 107 & 2.91 & 0.889 \\
\hline 8 & 1.54 & 0.744 & 58 & 2.98 & 0.982 & 108 & 2.63 & 0.776 \\
\hline 9 & 3.58 & 0.743 & 59 & 1.81 & 0.873 & 109 & 1.82 & 0.966 \\
\hline 10 & 3.29 & 0.725 & 60 & 3.22 & 0.74 & 110 & 2.82 & 0.884 \\
\hline 11 & 2.79 & 0.985 & 61 & 0.81 & 0.727 & 111 & 0.44 & 0.714 \\
\hline 12 & 3.37 & 0.738 & 62 & 3.29 & 0.84 & 112 & 3.43 & 0.84 \\
\hline 13 & 1.82 & 0.937 & 63 & 2.83 & 0.808 & 113 & 1.71 & 0.796 \\
\hline 14 & 1.78 & 0.975 & 64 & 2.96 & 0.836 & 114 & 3.2 & 0.86 \\
\hline 15 & 2.34 & 0.857 & 65 & 3.39 & 0.679 & 115 & 3.15 & 0.985 \\
\hline 16 & 2.71 & 1.046 & 66 & 1.65 & 0.821 & 116 & 2.37 & 1 \\
\hline 17 & 3.17 & 0.813 & 67 & 2.76 & 0.886 & 117 & 3.78 & 0.508 \\
\hline 18 & 2.57 & 0.913 & 68 & 3.03 & 1.095 & 118 & 1.8 & 1.011 \\
\hline 19 & 2.55 & 0.799 & 69 & 1.85 & 0.97 & 119 & 2.57 & 0.714 \\
\hline 20 & 3.34 & 0.882 & 70 & 2.17 & 0.928 & 120 & 2.31 & 1.06 \\
\hline 21 & 3.24 & 0.788 & 71 & 1.65 & 0.964 & 121 & 3.44 & 0.631 \\
\hline 22 & 1.81 & 1.026 & 72 & 1.7 & 0.996 & 122 & 3.61 & 0.658 \\
\hline 23 & 1.99 & 0.975 & 73 & 1.82 & 0.825 & 123 & 1.64 & 0.867 \\
\hline 24 & 1.61 & 0.798 & 74 & 1.79 & 0.926 & 124 & 1.55 & 0.889 \\
\hline 25 & 1.36 & 0.586 & 75 & 1.66 & 0.884 & 125 & 1.27 & 0.708 \\
\hline 26 & 2.22 & 0.936 & 76 & 2 & 0.931 & 126 & 2.57 & 1.091 \\
\hline 27 & 2.15 & 0.986 & 77 & 0.83 & 0.817 & 127 & 2.03 & 0.967 \\
\hline 28 & 2.31 & 0.933 & 78 & 0.97 & 0.975 & 128 & 2.7 & 1.021 \\
\hline 29 & 1.94 & 0.896 & 79 & 1.84 & 1.09 & 129 & 1.99 & 1.02 \\
\hline 30 & 0.97 & 0.908 & 80 & 0.99 & 0.865 & 130 & 2.36 & 1.065 \\
\hline 31 & 3.13 & 0.795 & 81 & 2.08 & 0.951 & 131 & 2.31 & 1.044 \\
\hline 32 & 2.29 & 1.101 & 82 & 0.66 & 0.834 & 132 & 2.65 & 1.108 \\
\hline 33 & 1.72 & 0.993 & 83 & 2.97 & 0.9 & 133 & 3.23 & 0.771 \\
\hline 34 & 2.81 & 0.985 & 84 & 3.46 & 0.789 & 134 & 2.96 & 0.881 \\
\hline 35 & 1.65 & 0.917 & 85 & 2.32 & 1.002 & 135 & 2.52 & 1.074 \\
\hline 36 & 2.06 & 0.918 & 86 & 1.8 & 0.821 & 136 & 1.92 & 0.92 \\
\hline 37 & 2.17 & 1.068 & 87 & 2.66 & 1.042 & 137 & 2.1 & 1.013 \\
\hline 38 & 2.19 & 1.041 & 88 & 2.15 & 0.88 & 138 & 3.01 & 0.996 \\
\hline 39 & 1.8 & 0.819 & 89 & 2.1 & 1.035 & 139 & 1.92 & 0.914 \\
\hline 40 & 1.6 & 0.725 & 90 & 1.7 & 0.898 & 140 & 1.61 & 0.638 \\
\hline 41 & 1.68 & 0.739 & 91 & 2.33 & 0.84 & 141 & 1.09 & 0.889 \\
\hline 42 & 2.42 & 0.994 & 92 & 3.59 & 0.619 & 142 & 2.34 & 0.97 \\
\hline 43 & 1.85 & 0.981 & 93 & 1.8 & 0.852 & 143 & 2.81 & 1.064 \\
\hline 44 & 2.64 & 1.013 & 94 & 2.86 & 0.89 & 144 & 3.32 & 0.866 \\
\hline 45 & 2.27 & 0.905 & 95 & 1.92 & 0.94 & & & \\
\hline 46 & 1.75 & 0.986 & 96 & 1.19 & 0.833 & & & \\
\hline 47 & 1.85 & 0.9 & 97 & 3.15 & 0.742 & & & \\
\hline 48 & 0.51 & 0.765 & 98 & 3.53 & 0.764 & & & \\
\hline 49 & 2.38 & 0.885 & 99 & 1.9 & 0.929 & & & \\
\hline 50 & 1.87 & 0.953 & 100 & 2.83 & 0.96 & & & \\
\hline
\end{tabular}




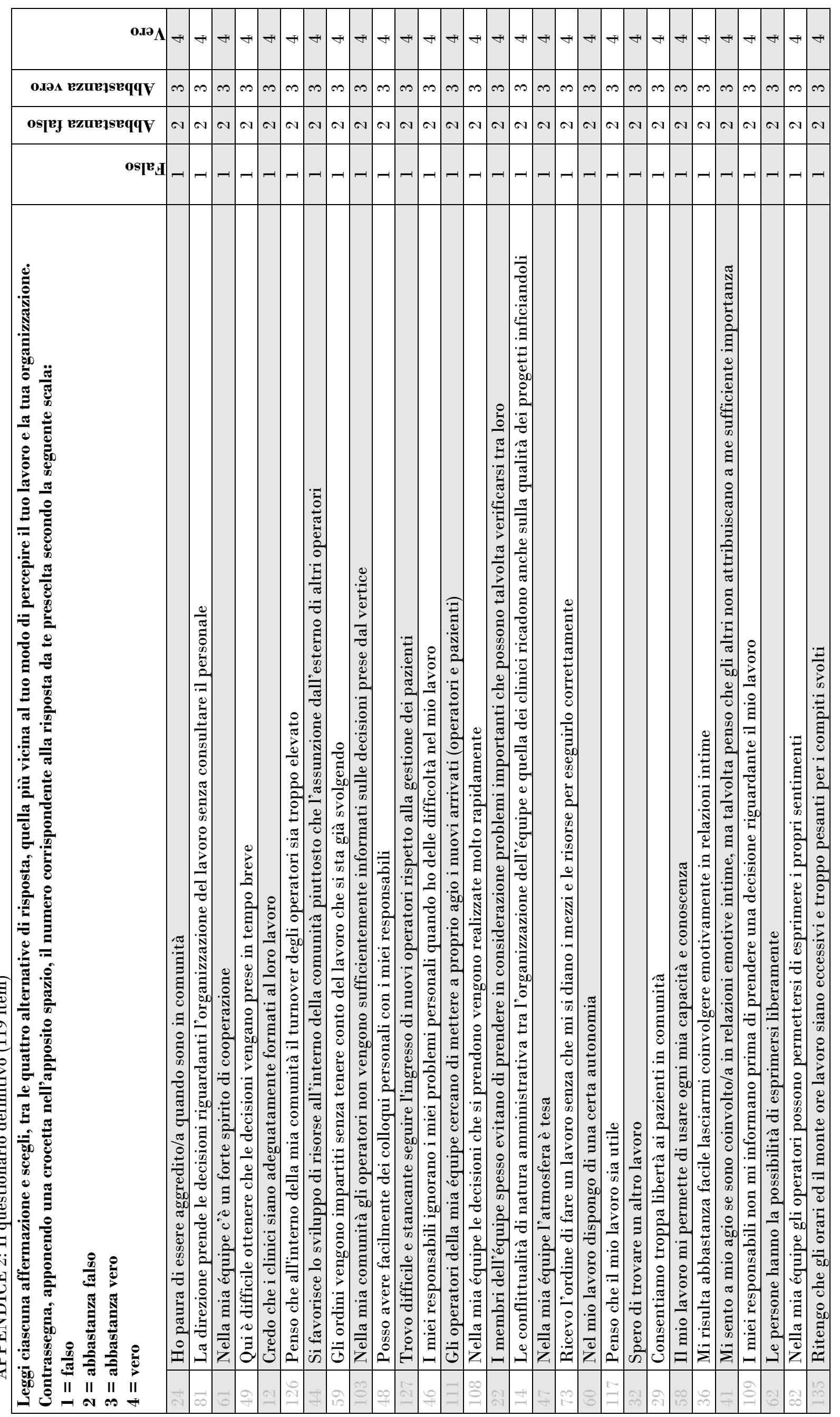




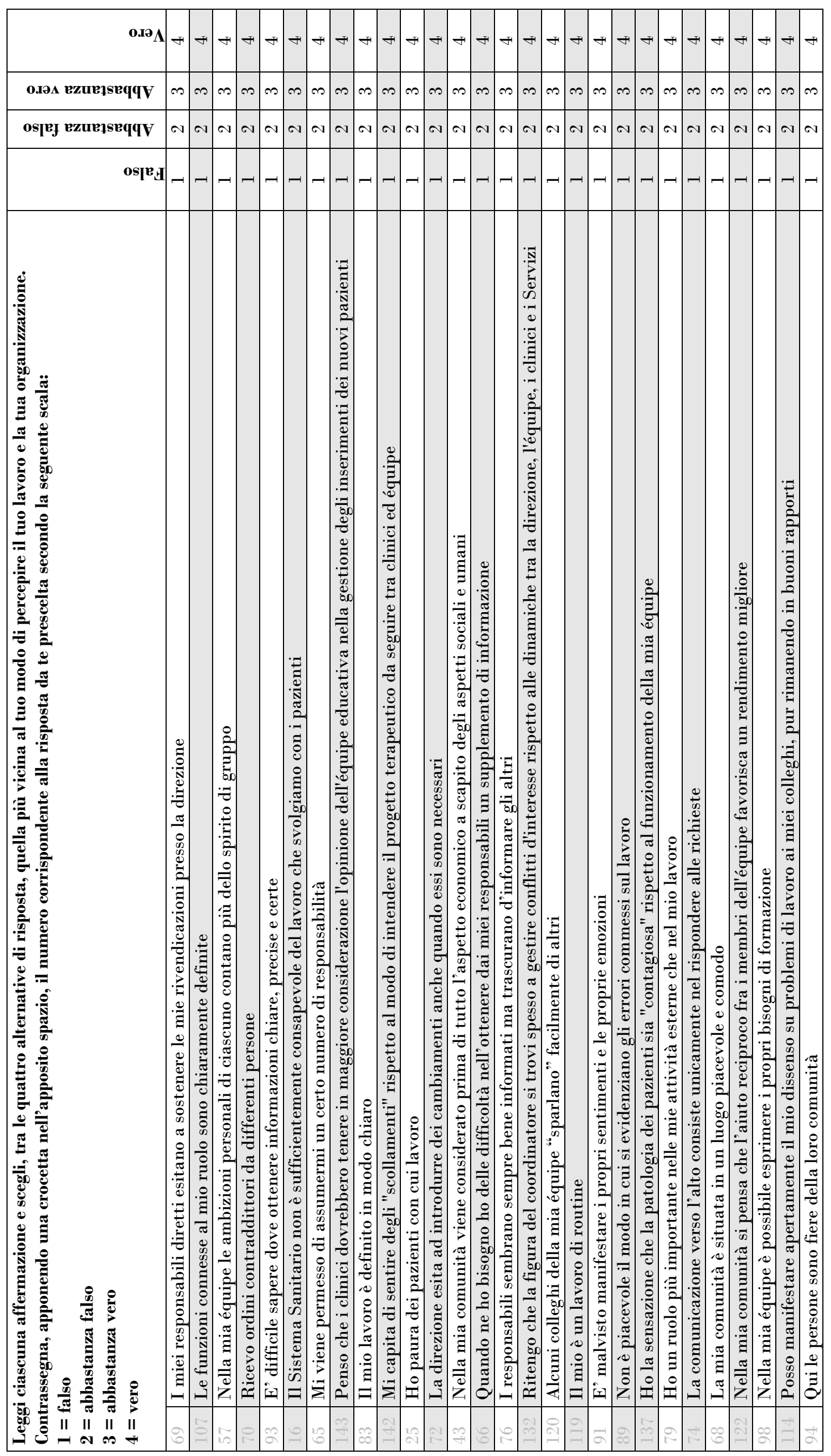




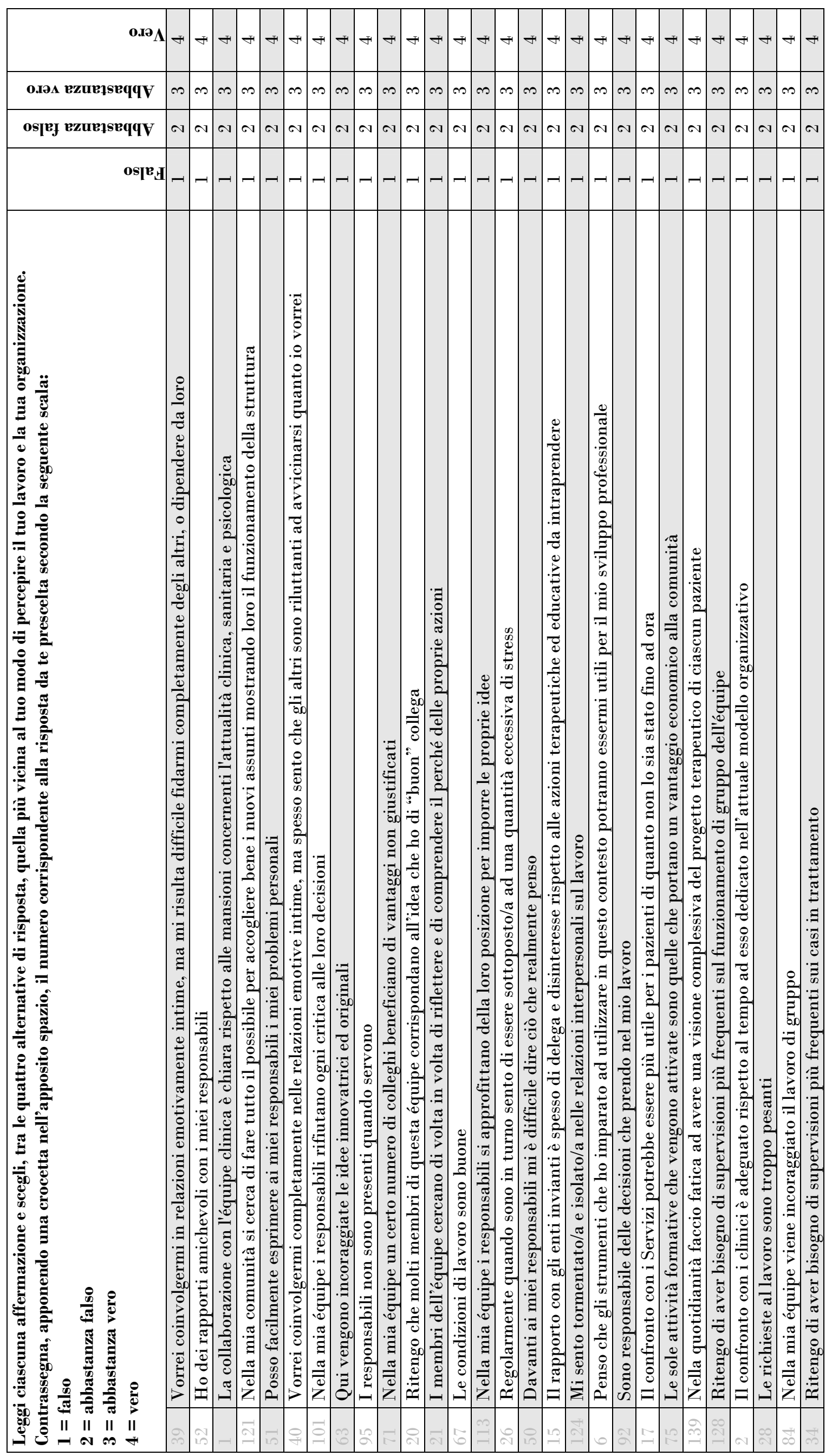




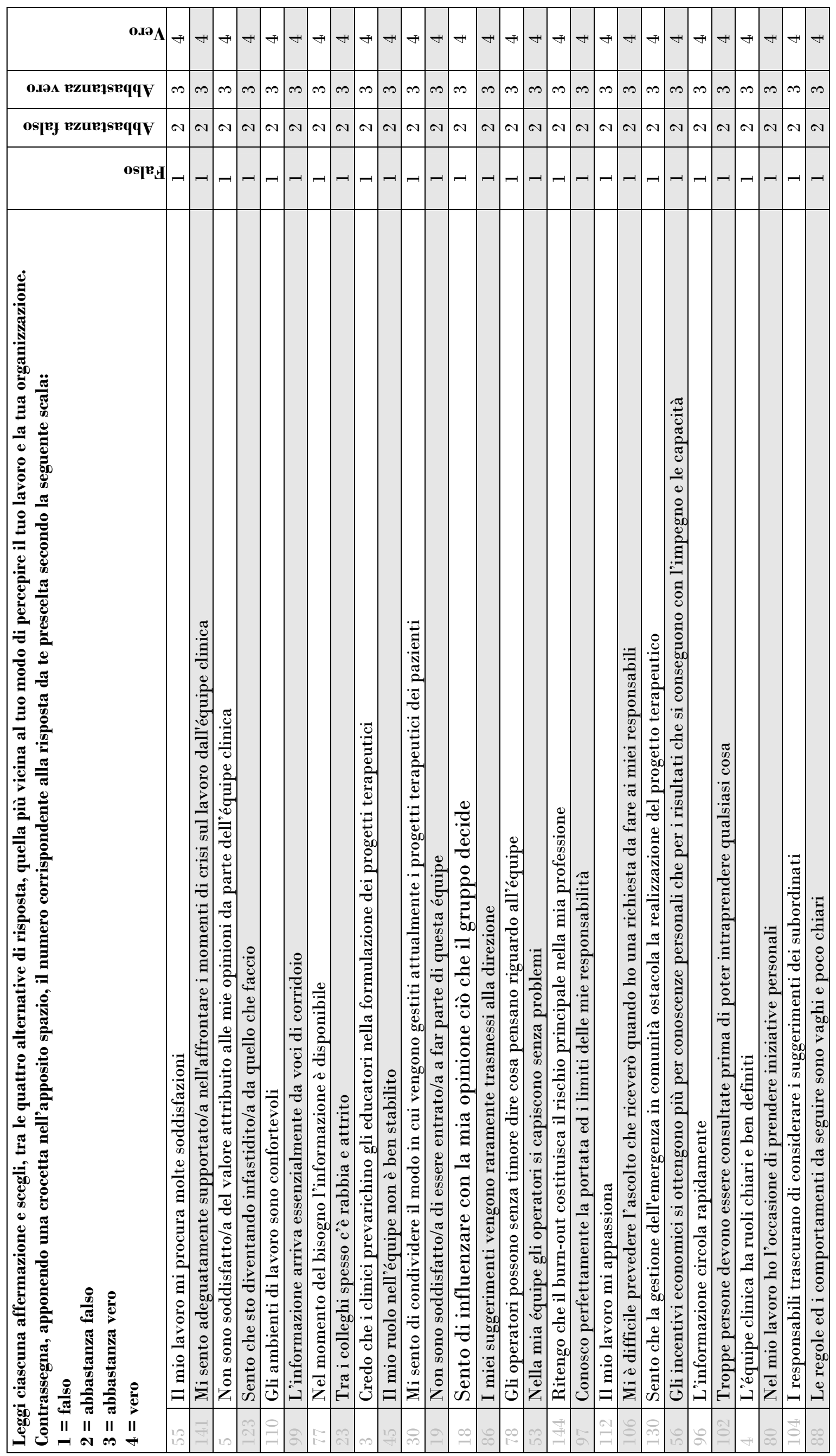




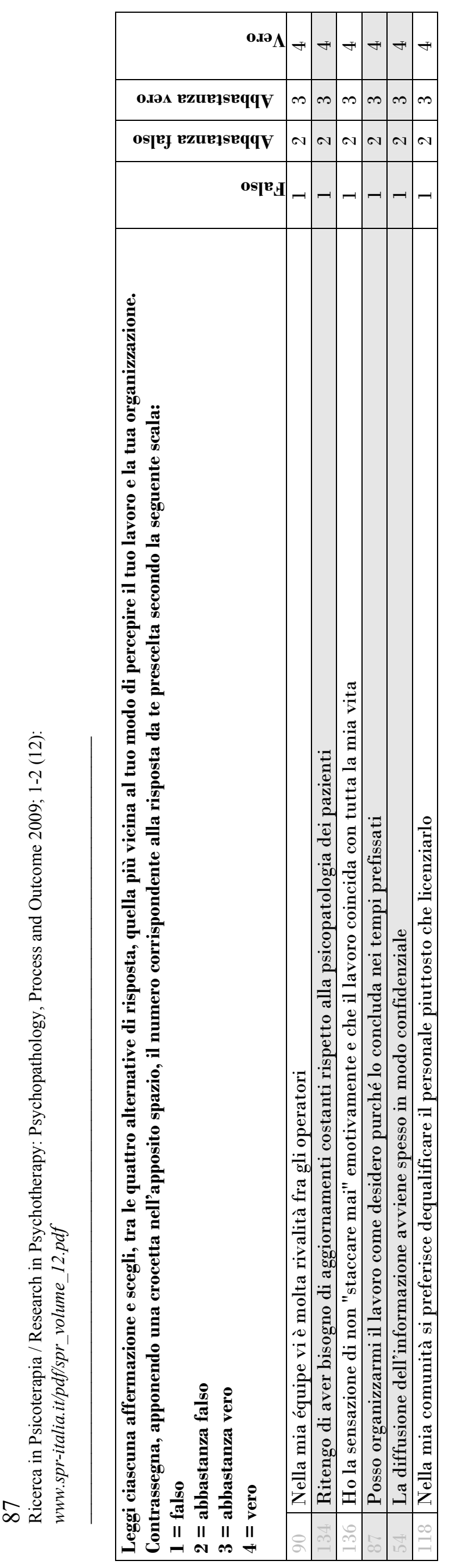

